\title{
INTEGRATION OF THREE DIMENSIONAL DATA FROM UNKOWN ORIGIN ON THE EXAMPLE OF A TRADITIONAL RESIDENTIAL BUILDING IN AL-BALAD, JEDDAH, SAUDI ARABIA
}

\author{
U. Herbig ${ }^{\text {a }}$ I. Mayer ${ }^{\text {a }}$, H. Mortada ${ }^{\text {b }}$, S. Rasztovits ${ }^{\text {c }}$ \\ a Department for History of Architecture and Building Archaeology, Vienna University of Technology, Austria - \\ baugeschichte@e251.tuwien.ac.at \\ ${ }^{\text {b}}$ Department of Architecture, Faculty of Environmental Design, King Abdul Aziz University, Jeddah, Saudi Arabia - \\ hmortada@kau.edu.sa \\ ${ }^{\mathrm{c}}$ Department of Geodesy and Geoinformation, Vienna University of Technology, Austria - \\ tpe_scathe@gmx.at
}

Commission V, WG V/2

KEY WORDS: 3D Laser Scanning, data merging, Saudi Arabia

\begin{abstract}
:
3D Laser scanning technology gained more and more importance for the recording and documentation of architectural heritage. Especially for the survey of heterogeneous surfaces and complex structures it is a fast and reliable option for survey and so appreciated sources for research in architecture. Therefore the integration of laser scanning as a part of the building survey became a kind of standard procedure for objects of different scale, shape, age and origin.

In some cases more than one team records an object with different devices using altering approaches. For example a client provides existing data from a part of the object that can't be accessed anymore, but is important to be integrated into the overall survey. The merging of the datasets may become challenging, especially if one survey is not documented in detail, in particular when it comes to the quality of the result.

For a research about the traditional architecture of Saudi Arabia a building in the historic part of Jeddah has been surveyed in detail by a team of researchers of the Vienna University of Technology. Within this frame a workshop for students of the King Abdul Aziz about building archaeological research has been conducted. A part of the results consists of two sets of laserscan data, recorded with different laser scanners. Using these data a possible approach for the registration of scan data from different and/or unknown provenance has been developed which will be outlined in this paper.
\end{abstract}

\section{INTRODUCTION}

The traditional architecture of Saudi Arabia is one of the unknown parts of the world's architectural heritage and therefore not documented yet. Within the frame of a research initiative of the Higher Ministry for Education Saudi Arabia a cooperation between King Abdul Aziz University (KAU), Jeddah and the Vienna University of Technology (VUT) was established in 2011 and with the research project Documentation and Analysis of Traditional Architecture of Saudi Arabia (DATARSA). The aim of the project is the research of the traditional built environment following a holistic approach. Therefore studies have been done in scales from urban structures to detailed analysis of the materials and construction of objects.

In autumn 2011 the project started with the joint workshop Traditional Architecture Recorded by Means of Building Archaeology in Saudi Arabia (TRABASA) in Jeddah. The aim of the cooperational works was the transfer of knowhow about details of the historic buildings in Jeddah from KAU side and methods for the building archaeological research from the other side. Within this frame the research of the architecture in $\mathrm{Al}$ Balad, in the historical part of Jeddah has been conducted. In interdisciplinary teams (architects, geodesists, landscape architects and an anthropologist) different aspects of the traditional built environment have been investigated.

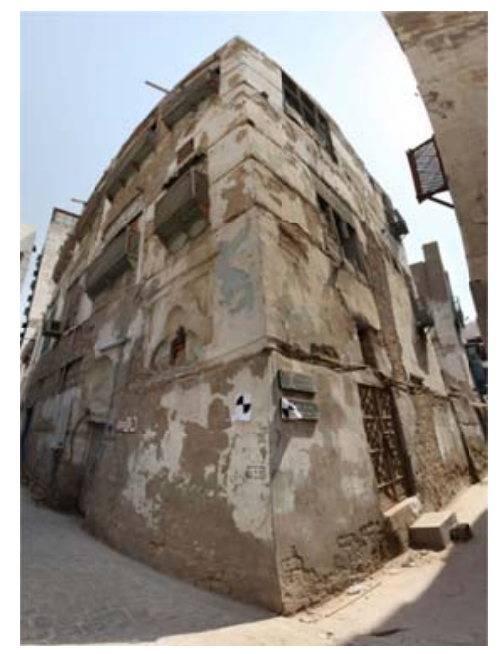

Figure 1. Residential building Al Nawar, in the historic centre of Jeddah, KAU; 4 images stitched (C) Irmengard Mayer

One important part was the documentation of an ordinary residential building in the heart of $\mathrm{Al}$ Balad as starting point of a building archaeological survey. In theoretical and practical training sessions, staff and students of KAU have been introduced to recording and documentation methodologies for historic architecture used by the VUT team. Thereafter the work 
of building research in the house was done in intensive collaborative work of KAU students and the VUT researchers in a joint campaign. The building survey covers all parts of the object compiled in plans, a catalogue of openings, and a room book mapping each wall with its materials and damages. This material will be the base for the restoration of the building.

\section{DATA AQUISITION}

Beside further documentation (e.g. photo documentation, room book, catalogue of features etc.) a three dimensional survey with the terrestrial 3D-Image-Scanner Z+F Imager 5006i supported by tachometric survey with a motorized Leica TCRM 1203+ was done. In addition to this 3d-survey some scans have been done with a Leica Scan Station C10by KAU.

The tachometric survey started with polygon tracks in and around the surveyed residential building as base for the geodetic integration of all other collected data. Starting from this polygon tracks all necessary control points for the later 3D laser scanning with the Z+F Imager 5006i and rectified images were surveyed. The recording of the polygon track and the control points on site was done in simple sketches made by hand like it is common practice. The horizontal sections in all floors as well as one cross section were measured with the total station using a special software (TOTAL - Tachometric Object-Oriented Semi Automated Laser Measurement developed by the Ruhr University Bochum, Germany). Along the longitudinal section all rooms and facades have been scanned with the $\mathrm{Z}+\mathrm{F}$ Imager $5006 i$.

A team from KAU surveyed the southern staircase which was hardly to survey by total station due to the very complex and narrow situation. The control points used for the survey by KAU with a Leica Scan Station differed from the control points observed by the total station. The data were handed over to VUT in the Leica proprietary format. Since the VUT does not own a Leica Scan Station and the associated software it was necessary to bring the data somehow into a readable format. This was done with a one-week test license.



Figure 2. Point cloud of Al Nawar house, surveyed with Z+F Imager 5006i (C) VUT

\section{PRECONDITIONS AND DATA PREPERATION}

Thus, each scanning company has its proprietary file format, first all single TLS scans has to be transformed into a common data format like ascii(*.xyz). After the export, the installed targets has to be identified inside each single point cloud. For each scan at least three targets are absolutely necessary for a registration. The more targets are identified the better the result of the registration will be. A target list for each point cloud has to be exported into an ascii-file (*.xyz). If the target list surveyed by total station (ascii-file, ${ }^{*}$.xyz) and one scan-target list are named correctly an automatic computation is possible.

\section{THE APPROACH FOR TRABASA}

Since the obtained TLS and total station data derive from different instruments surveyed by different teams, the merge of all these data was necessary. As mentioned above control points (small black/white targets) were installed at the walls of all rooms for the resampling of rectified images. These control points were also used for the registration of the TLS data. Within a local coordinate system a polygon track was surveyed with a total station and the control points were measured spherical. When performing a single scan (e.g. in the corner of one room) only a subset of all measured control points is visible (Figure 1). The documentation of the control points was done in simple sketches made by different people with different approach and understanding of the importance of a sketch. This resulted in drawings of significantly deviating quality and sometimes inconsistency (identification problems, ambiguities, etc.). This caused many problems at the computation of the absolute orientation of the single TLS scans. In order to avoid these problems a full automatic approach was implemented by considering geometric information between control points, aiming to identify corresponding control points deriving from a geodetical survey or other scans.

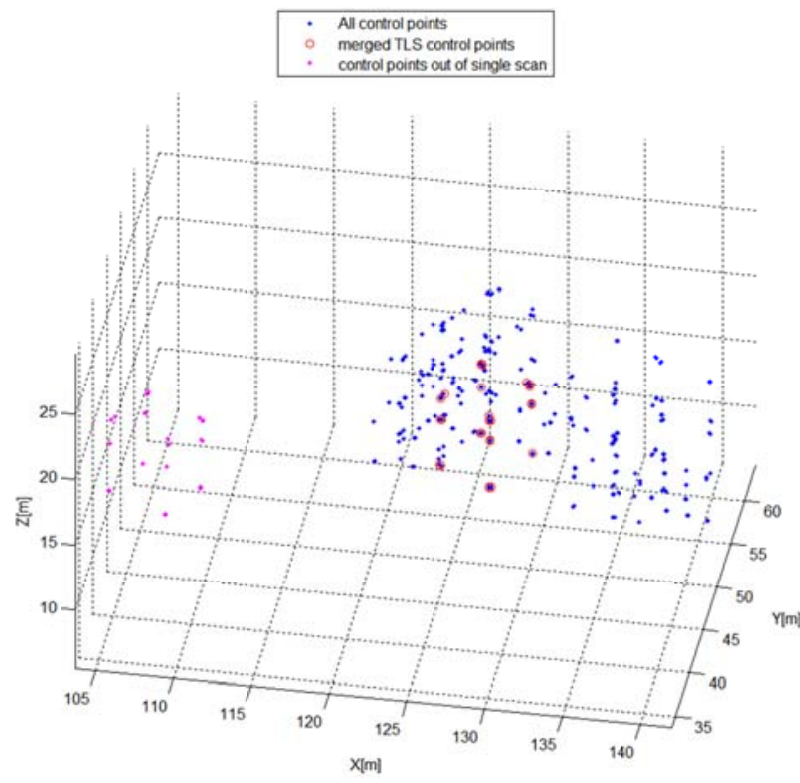

Figure 3. Blue - control point coordinates surveyed with total sitation.

Magenta - visible control points in one single scan, to be registered.

Red - automatically aligned TLS control points onto the control points surveyred with total station. 
Figure 3 is stating the problem. The unknown transformation between a single TLS scan and the realized coordinate system needs to be estimated. In order to achieve the orientation of a single TLS scan to the total station data, the estimation of transformation parameters needs to be performed. The 3D similarity transformation between two given corresponding point sets (A, B) is given by (Eq.1)[Kraus].

$$
\mathrm{A}=\mathrm{m} * \mathrm{R} * \mathrm{~B}+\mathrm{T}
$$

$$
\text { where } \quad \begin{aligned}
& \mathrm{m}=\text { a scaling factor, } \\
& \mathrm{R}=\text { the rotation matrix } \\
& \mathrm{T}=\text { the vector of shift. }
\end{aligned}
$$

Considering the euclidean 3D space, (Eq.1) contains seven unknown parameters. They can be estimated, if a minimum number of three non collinear point correspondences are present.

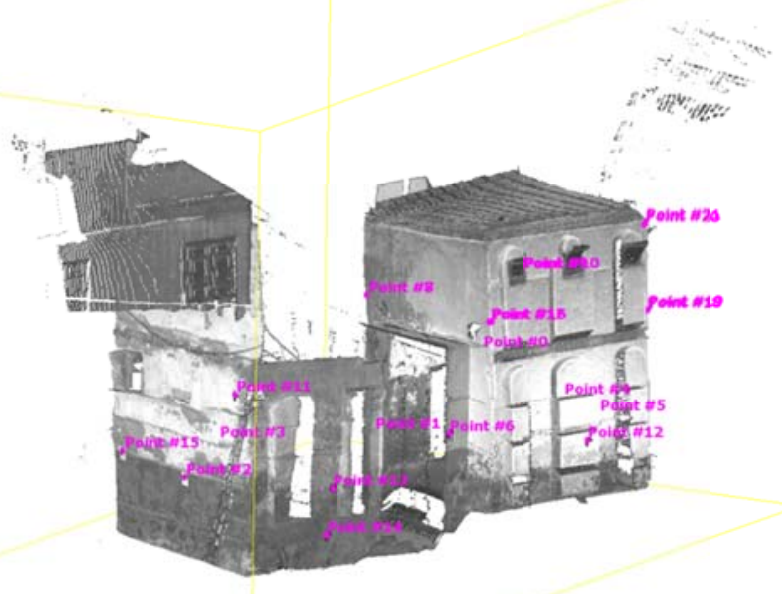

Figure 4.Identification of all visible targets in one single scan of a two-storey room and the adjacent court yard

With the help of the open source software CloudCompare the targets were selected in each single TLS scan and the coordinates of these targets readout into a text file (compare Figure 4). The coordinates of these scans are in the scanner own coordinate system.

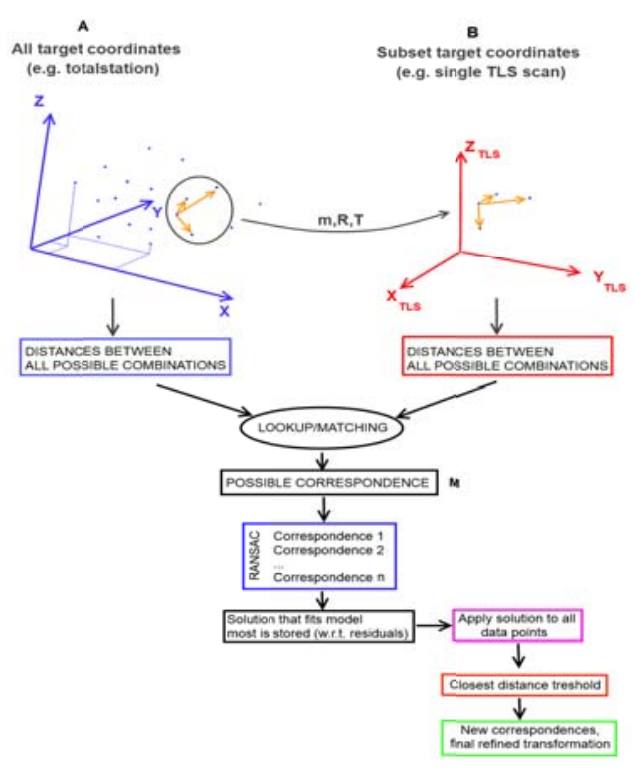

Figure 5. Description of the algorithm
Figure 5 is describing the algorithm of automatically match point correspondences in 3D space. An euclidean 3D distance between pairs of all possible combinations is computed for both coordinate lists (A, B). The size of the resulting distance matrix is linearly scaled by the amount of given data points. Via a simple lookup of corresponding distances ( $\mathrm{B}$ in $\mathrm{A}$ ) with a certain threshold amount $\varepsilon_{t}$, it is possible to identify possible corresponding rows inside both distance matrices. We chose the factor to be smaller than $2 * \sigma_{0}^{2}>\varepsilon_{t}$, where $\sigma_{0}^{2}$ is the a priori standard deviation of our TLS instrument (in our case $3 \mathrm{~mm}$ ). Possible correspondences are stored in the matching matrix (M). With those possible correspondences and the mathematical model of the similarity transformation, a solution of the transformation parameters, as well as residual vectors, can be computed by adjusting all observations. By selecting a minimum number of three corresponding random sample matches inside the matching matrix (M), a solution of the unknown transformation parameters can be estimated. Because the functional model between the parameters and the observations is nonlinear, we need a linearization of the functional model by a taylor series. Because high angle rotations between both coordinate frames can occur, it is necessary to start the adjustment with good initial estimates of the unknown parameters. Therefore we used a quaternion transformation - its description is given in [Horn]. Due to distance ambiguities inside the matching process, we performed a RANSAC algorithm, by considering the remaining points that are not involved in the computation of the initial solution of the parameters.

The points inside the TLS scan (B) can now be transformed to the coordinate system (A) with the possible initial solution. As mentioned above, only the minimum and necessary amount of points (three R3 points) was used for the computation of the unknown transformation parameters. Thus, points inside (A) and (B) are now in a common coordinate frame and it is now possible to find additional points that are close to each other. An additional correspondence is assumed, if the distance between points inside (A) and (B) is under a certain threshold value $\left(\varepsilon_{D}\right)$. In our case, we chose $\varepsilon_{D}$ to be $3 * \varepsilon_{t}$.

Considering those additional observations inside a least squares adjustment, the initial transformation parameters can be refined. The refinements respective parameter updates (3) can be computed considering the over-dettermined equation system (2), aiming to minimize the sum of squared observation corrections $\left(\sum v^{\prime} P v \rightarrow \min \right)$.

$$
A x=l+v
$$

where $\quad A=$ jacobi matrix,

$$
\begin{aligned}
& \mathrm{X}=\text { vector of unknowns } \\
& \mathrm{l}=\text { observation vector } \\
& \mathrm{v}=\text { correction vector }
\end{aligned}
$$$$
\hat{x}=\left(A^{\prime} * P * A\right)^{-1} * A^{\prime} * P * l=N^{-1} * A^{\prime} * P * l
$$

where $\quad \mathrm{N}^{\wedge}-1$.. inverted normal-matrix

The accuracy of individual transformation parameters (5) can be estimated via the unit weight coefficient (4) and its diagonal elements inside the inverted normal-equation matrix $\mathrm{N}\left(Q_{x x}\right)$ inside (3). Considering more observations, the redundancy $(n-u)$ increases (enlargement of three per observed point), causing a reduction of the unit weight coefficient respective the accuracy of each transformation parameter. 


$$
\hat{\sigma}_{0}=\sqrt{\frac{v^{\prime} * P * v}{n-u}}
$$

where $\quad \mathrm{v}=$ correction vector

$\mathrm{P}=$ Weighting matrix

$\mathrm{n}=$ number of observations

$\mathrm{u}=$ number of unknowns

$$
q_{x k}=\hat{\sigma}_{0} * \sqrt{q_{k k}}
$$

where

qxk = mean error of one single unknown

qkk = diagonal element of the inverted normal matrix

Selected target coordinates (from a single scan), that were not matched to the list of total station coordinates, are appended to the total-station coordinate list (A). When applying the same algorithmic (e.g. the target coordinates of an additional TLS scan), those additional points can support the estimation of the transformation parameters of further scans.

As mentioned above, the approach tries to find identical distances in two lists of coordinates. Therefore, it is necessary that the distribution of distances inside the two coordinate lists are nearly unique. Considering (e.g.) vertices of a cube, this approach would run fail in order to the distance ambiguity and the resulting non-unique solution of the transformation parameters. In practice as well as the handled data inside this project, the distance pattern of the target points was nearly unique per each scan.

\section{RESULTS AND SIMULATION}

This chapter shows some achieved results that were obtained from the approach mentioned above .

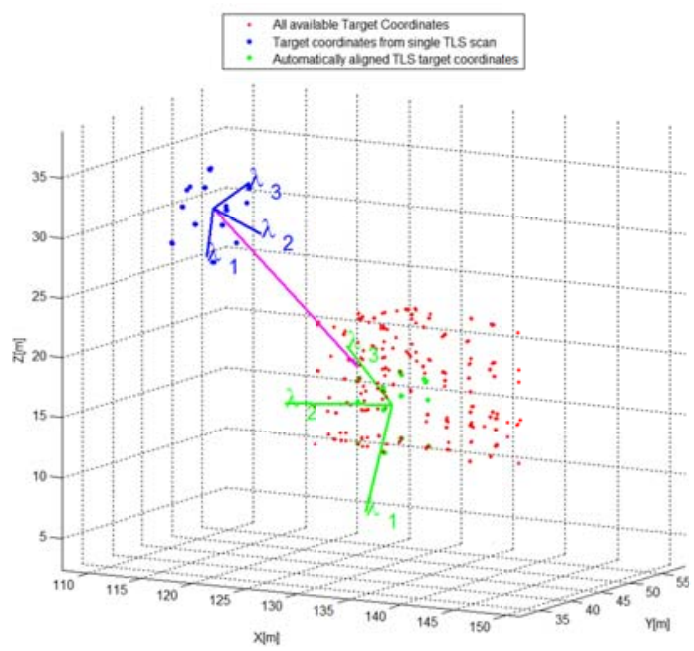

Figure 6.Automatically aligned TLS points

Starting with given manually selected control points (originated by a single TLS scan) (Fig. 6 blue dots), the final transformation parameters are computed in automatically manner. The unknown transformation parameters (Fig. 6 represented by the magenta line), a matching/correspondence list as well as residual vectors of a single scan with reference to the total station coordinate list are computed.

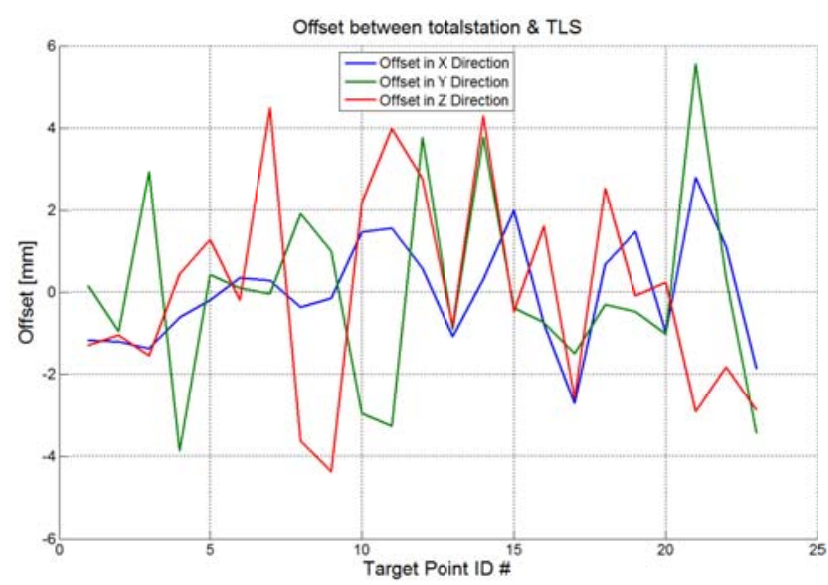

Figure 7. Computed components; of the residual vectors after applying the final (adjusted)

transformation between corresponding point pairs.

After the estimation of the unknown transformation parameters completed, final residuals of point correspondences can be computed. Figure 7 shows the coordinate differences among the three coordinate axis for each pointt correspondence.

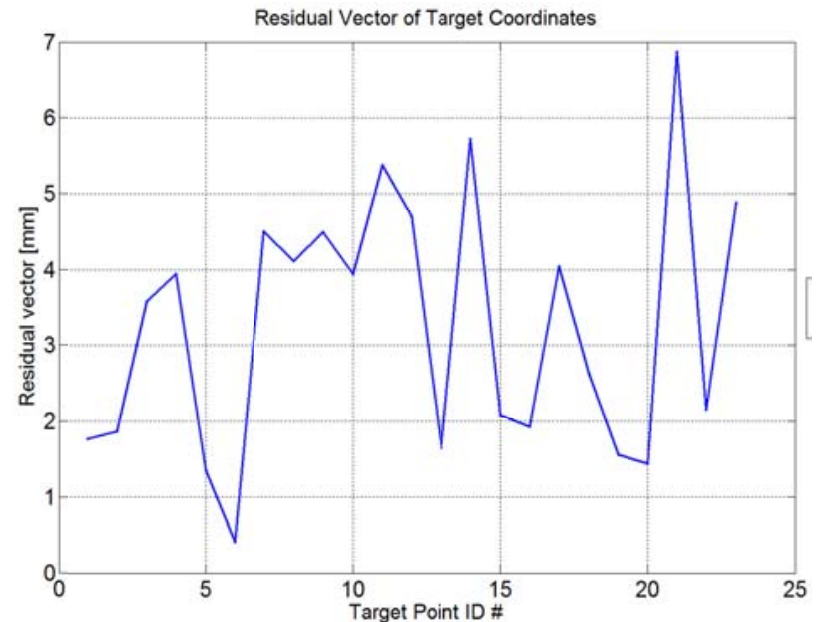

Figure 8. Length of the residual vector between corresponding points

In order to check the described algorithm in a more detailed way, we designed some test-cases. Therefore, we chose some randomly selected points out of the whole tachymeter list. Those points are then transformed by a random shift vector and a rotation matrix. To achieve real conditions, we added some normal distributed noise to the selected coordinates. This was done by applying a standard-deviation of $3[\mathrm{~mm}]$ per coordinate axis and a linear scale factor [ppm], that was correlated by the distance between the center of gravity (with reference to the selected points) and each single point. Figure 8 shows some test scenarios by considering five random point correspondences. 

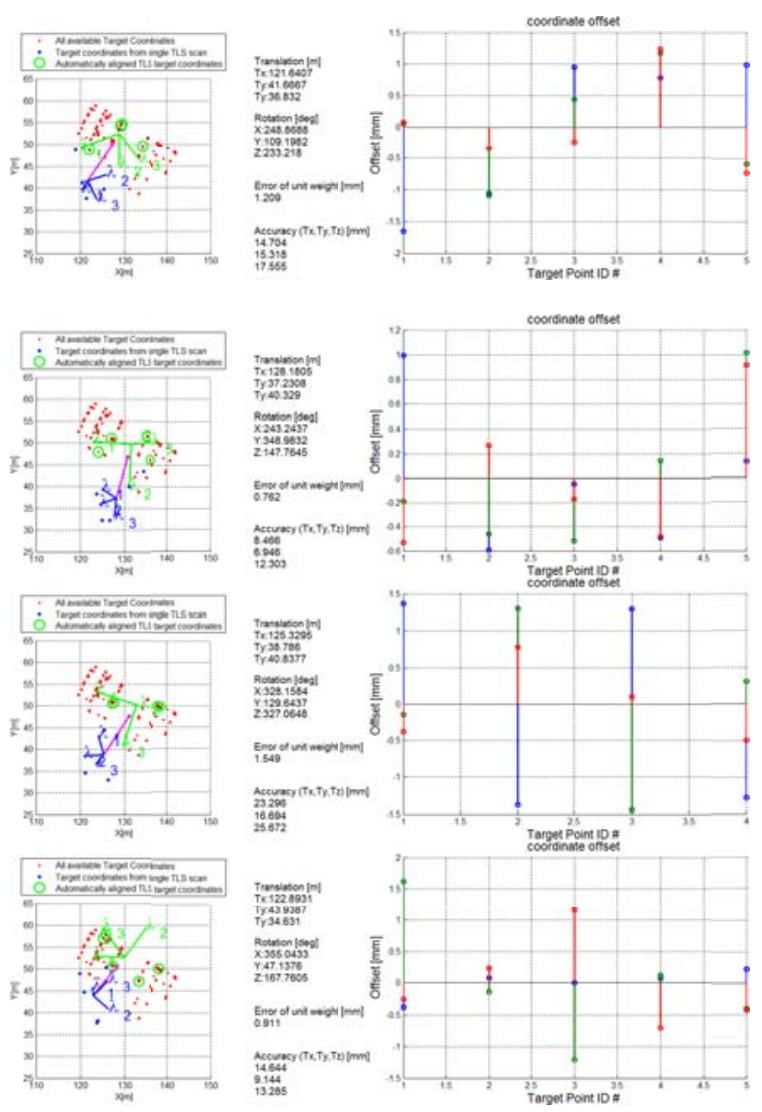

Figure 9. Test-cases of randomly selected coordinate subsets

\section{CONCLUSION}

The final implementation of the algorithmic should work also with target points with different scale (e.g. relative orientated photogrammetric point clouds). Instead of distances, relative angles and distance ratios can be used for the identification process.

The described approach (chapter 4) estimates the unknown transformation parameters between single TLS target coordinates and totalstation target coordinates. It works pretty well among the considered data inside this project and has potential to be realized in a standalone application or as a plugin in already existing software tools to support further processing.

\section{Acknowledgement}

This work was financed by the Kingdom of Saudi-Arabia, Prince Abdul Aziz University in Jeddah.

For the building survey on the Al Nawar house we want express special acknowledgements for direct involvement in the work on this distinctive building. A special thank has to be expressed to Eng. Sami Nawar as the representative of the municipality, and also as the representative of the owners of the Al Nawar house for his tremendous support in the work for the building survey of this special example of Jeddah's architectural history.

\section{References}

Bokhari, Abdulla Y., 1983. Conservation in the Historic District of Jeddah. In Adaptive Reuse: Integrating Traditional Areas into the Modern Urban Fabric. Margaret Bentley Sevcenko, (ed). Cambridge, Mass.: MIT Laboratory of Architecture and Planning, USA.

Harrow, L., Larkin, C., 1984. City of the Red Sea, David Howell's Jiddah, Scorpion Publishing Ltd., London, GB.

Herbig, U., 2012. Interdisciplinary Approaches to the Built Heritage and their Role for World Heritage Sites Research \& Heritage: Researchpapers on Architectural Heritage/ The Saudi Commission for Tourism and Antiquities, Dammam, 1434H, 2012, page 263-285

Herbig, U., Jäger-Klein, C., Mayer, I., Mortada, H., StyhlerAydın, G., 2013. TRABASA - Traditional Architecture recorded by Means of Building Archaeology in Saudi Arabia: Workshop in Jeddah. In: International Archives of the Photogrammetry, Remote Sensing and Spatial Information Sciences, Volume XL-5/W2, 2013, XXIV International CIPA Symposium, 2-6 September 2013, Strasbourg, France

King, G. 1998.The Traditional Architecture of Saudi Arabia, L.B. Tauris\& Co Ltd., London, GB.

Kraus K., 2004. Photogrammetrie Band 1.Geometrische Informationen aus Photographien und Laserscanneraufnahmen, de Gruyter, Berlin, Germany.

Horn B. K.P., 1987. Closed-form solution of absolute orientation using unit quaternions. In: Journal of the Optical Society of America, Vol.4, 1987, Washington D.C, page 629642

Sieghartsleitner, A., 2010. TraditionelleBauformenimWesten Saudi Arabiens, Diploma Thesis, Vienna University of Technology, Department for History of Architecture and Building Archaeology, Vienna, Austria.

Styhler-Aydın, G., Mayer, I., 2012. 3D Laser Measurement as Part of an Integrative Building Survey for the Recording of Built Heritage; Invited Paper at: 17th International Conference on Cultural Heritage and New Technologies, Vienna, 05.11.2012 07.11.2012, in print

Toubekis, G., Mayer, I., Döring-Williams, M., Maeda, K., Yamauchi, K., Taniguchi, Y., Morimoto, S., Petzet, M., Jarke, M., Jansen, M., 2009. Preservation and management of the UNESCO world heritage site of Bamiyan: Laser scan documentation and virtual reconstruction of the destroyed Buddha figures and the archaeological remains.In: Takase, Yutaka (Ed.): 'Digital Documentation, Interpretation \& Presentation of Cultural Heritage" , CIPA Symposium , 11 - 15 Oct. 2009 in Kyoto, Japan 